\title{
Prevalence, determinants, and characteristics of extemporaneous compounding in Jordanian pharmacies
}

\author{
Hatim S. AlKhatib ${ }^{1}$, Sara Jalouqa', Nour Maraqa ${ }^{1}$, Anna Ratka ${ }^{2}$, Eman Elayeh $^{3}$ and Suha Al Muhaissen ${ }^{*}$ (D)
}

\begin{abstract}
Background: Pharmaceutical compounding is an essential component in pharmacy practice allowing pharmacists to provide dosage forms or strengths that are commercially unavailable. Medications compounded for patientspecific needs contribute to personalized medicine. Extemporaneous compounding provided by pharmacies overcomes the market shortage of these therapeutic products. The aim of this study is to investigate and characterize the prevalence, characteristics, and determinants of extemporaneous compounding in Jordanian pharmacies.
\end{abstract}

Methods: This study was based on a cross-sectional questionnaire and included 431 randomly selected pharmacies in the twelve governorates of the country. Data were collected via face to face interviews of pharmacists who voluntarily and verbally responded to the questions.

Results: Results revealed that 223 (51.7\%) of the surveyed pharmacies practiced extemporaneous compounding. The main reason for not providing extemporaneous compounding services was lack of prescription orders for compounded preparations (53.8\%). The second reason was lack of the equipment and supplies necessary for compounding (24.4\%). Extemporaneous compounding prescriptions were mainly issued by dermatologists (98.2\%); dermatological indication was the most common of all extemporaneous compounded prescriptions. The main reason for requesting compounded medications was the lack of a commercially available product (87.9\%). The vast majority of the compounded dosage forms were creams (99.6) and ointments (91.5), followed by solutions (23.3\%). Only 5 (2.2\%) of the studied compounding pharmacies prepared sterile products. The major sources for compounding protocols were the physician order (94.2\%), and 'in-house' protocols (44.8\%). However, the main resource for estimating compounded medications expiration date was information based on pharmacist's experience (57.8\%) and the physician's order (53.4\%).

Conclusions: Extemporaneous compounding is a common element of pharmaceutical care. Topical preparations are the most commonly compounded products. Finding from this study suggest that there is a need for standardizing the compounded product formularies, product quality testing, and improving the consistency in estimation of an expiration date of compounded products.

Keywords: Extemporaneous compounding, Pharmacy practice, Dosage forms, Community pharmacy services, Hospital pharmacy, Pharmacists, Prescriptions, Pharmaceutical care, Jordan

\footnotetext{
* Correspondence: s.muhaissen@ju.edu.jo

1Department of Pharmaceutics and Pharmaceutical Technology, School of

Pharmacy, The University of Jordan, Queen Rania Street, Amman 11942,

Jordan

Full list of author information is available at the end of the article
}

(c) The Author(s). 2019 Open Access This article is distributed under the terms of the Creative Commons Attribution 4.0 International License (http://creativecommons.org/licenses/by/4.0/), which permits unrestricted use, distribution, and reproduction in any medium, provided you give appropriate credit to the original author(s) and the source, provide a link to the Creative Commons license, and indicate if changes were made. The Creative Commons Public Domain Dedication waiver (http://creativecommons.org/publicdomain/zero/1.0/) applies to the data made available in this article, unless otherwise stated. 


\section{Background}

Extemporaneous compounding refers to the practice of the preparation of a therapeutic product by mixing and combining pharmaceuticals agents for an individual patient in response to an identified need [1]. Extemporaneous compounding was traditionally used to fill most of the prescription orders submitted to pharmacists until the advent of pharmaceutical industry in the second half of the nineteenth century [2].

Nevertheless, extemporaneous compounding is still a relevant pharmaceutical service provided by many pharmacies worldwide [3-8]. The continued need for extemporaneous compounding in the age of pharmaceutical manufacturing is driven by the individualized patient care focusing on the therapeutic needs of patients with rare diseases [9], pediatric patients [10-12] and patients with requirements for special dosage forms, individualized dosing or active ingredients combination that are not provided commercially $[1,2,6]$. Moreover, from a professional point of view, compounding is a very unique attribute of a pharmacy practice that reflects on the professional status of pharmacists' provision for patient-specific healthcare needs [5].

Application of quality standards in extemporaneous compounding is still a major concern for regulatory agencies since poor quality of compounding practices can result in life-threatening contamination or in products that don't possess the required strength, quality, and purity [13]. In the United States, the process of extemporaneous compounding of sterile and nonsterile preparations in pharmacies, which are under the authority of the state boards of pharmacy, has been regulated, to varying extents, by the United States Pharmacopeia (USP) chapters $<795>$ Pharmaceutical Compounding - Nonsterile Preparations and $<797>$ Pharmaceutical Compounding - Sterile Preparations [14]. However, the safety and efficacy of compounded products are not clinically evaluated [15]. Extemporaneous compounding is performed in a considerable number of Jordanian pharmacies; however, the practice has never been assessed and characterized by any previous study. This study was designed to investigate the prevalence, characteristics, and determinants of extemporaneous compounding in Jordan. The findings from this study will improve the understanding of current extemporaneous compounding practices and provide information to guide efforts on developing the regulatory framework, educational curricula, and professional training needed to ensure safety and quality of compounded products and pharmaceutical care of patients.

\section{Methods}

\section{Data collection}

Data for this project were collected using a cross-sectional descriptive questionnaire. The questionnaire was constructed based on information collected from intense review of published relevant literature $[1,6,15,16]$. A 5section questionnaire was developed to obtain data from pharmacies to assess prevalence, frequency, and determinants of extemporaneous compounding (Additional file 1). The first section had thirteen items related to demographics of pharmacies and pharmacists' and descriptive data including: pharmacy name, address, and type, pharmacist gender, age, qualifications, and position, and the number of the pharmacy staff members; training provided by the pharmacy, and provision of compounding services. The second section described prescriptions characteristics and reasons for compounding. The third section assessed the indications, types, and dosage forms of compounded medications. The fourth section asked about tools and equipment available for compounding. The fifth section assessed the formulation properties; resources for compounding protocols and estimation of expiration date for compounded medications.

Administration of this questionnaire was based on a personal interview with the pharmacist on duty; the data collector asked the pharmacist the questions and filled the questionnaire. Four senior pharmacy students performed the interviews with the participants. All interviewers were trained by the principal investigator (PI) and two of the co-investigators. The training focused on communication skills with the pharmacy staff and on correct administration of the questionnaire. Moreover, the questionnaire was supplemented with a protocol with instructions. The interviewers were instructed to contact the PI in case of any uncertainty to ensure consistency in administration of the questionnaire.

The questionnaire was distributed to pharmacists from 431 randomly selected community and hospital pharmacies located in cities in the 12 governorates of Jordan. There were no exclusion criteria. The population frame was obtained as a list of all operating pharmacies (community and hospital) in the twelve governorates of Jordan. The list was provided by the Jordan Pharmacy Syndicate. Data were collected in February, 2018. Participating pharmacists were requested to sign an informed consent before responding to the questionnaire.

The data collection tool was evaluated by an expert in the field of pharmacy practice and piloting. A pilot test of the 26-item questionnaire was conducted in 30 pharmacies that were randomly-selected. The results from the pilot study were evaluated and used to modify the data collection tool before starting the main data collection period. Statistical confirmation of validity and reliability was achieved through the calculation of Cronbach' Alpha value which was found to be 0.907 indicating consistency of the tool. Sampling adequacy was also confirmed using Principal Components Analysis (PCA) with Kaiser-Meyer-Olkin (KMO) equal to 0.841 and a significant Bartlett's Test $(p=0.000)$. 
Table 1 Demographic characteristics of pharmacies participating in the Study

\begin{tabular}{ll}
\hline Characteristic & Number (\%) \\
\hline Pharmacies invited to participate in study & $444(100)$ \\
Pharmacies that participated in study & $431(97.1)$
\end{tabular}

\section{Pharmacy Type}

Hospital
Community
Chain
Independent
Pharmacist Gender ( $N=43$
Female
Male
Pharmacist Age, years
23-30
$30-40$
$40+$

Personnel

Pharmacist

Registered Pharmacy Assistant

Pharmacists' Qualification

Doctorate Degree

Master Degree

Bachelor Degree

Diploma Degree

Number of Pharmacists per Pharmacy

1
2
3
4
$\geq 5$

Number of Assistants per Pharmacy

$\begin{array}{ll}0 & 236(54.8) \\ 1 & 130(30.2) \\ 2 & 54(12.5) \\ \geq 3 & 11(2.5)\end{array}$

Number of Personnel per Pharmacy

0

1

2

$\geq 3$

Plan to Start Compounding Services $(N=208)$

$$
\text { Yes }
$$

No

Training on Compounding Provided in Pharmacy

For Pharmacists
Table 1 Demographic characteristics of pharmacies participating in the Study (Continued)

\begin{tabular}{ll}
\hline Characteristic & Number (\%) \\
\hline For Registered Assistants & $109(25.3)$ \\
\hline
\end{tabular}

To minimize social desirability bias, assurance was given to interviewees that discussions would be confidential and their responses would be anonymized. Collected data was stored with the corresponding author and further analysis was done anonymously.

\section{Statistical evaluation}

The sample size was calculated using compounded medications prevalence values (P) described in Buurma et al. [16] and Zaid et al. [6], $Z_{\alpha}=1.96$, and $d=0.05$. This calculation showed that 50 to 308 pharmacies were required to ensure reliable sample size to describe the compounded medication frequency and characteristics.

Categorical data were presented as frequency and percentages, while continuous data were presented as median and interquartile ranges.

Bivariate analysis was used to test the differences among the variables that affect the compounding of extemporaneous drug preparations. Chi-square or MannWhitney tests were used to detect differences between different determinants. Logistic regression was used to evaluate the strength of association of factors that significantly determine the characteristics of compounding in the bivariate analysis. All hypothesis testing was twosided. A $P$-value of $<0.05$ was considered significant. Data analysis was performed using SPSS $^{\curvearrowleft} 23.0$ (IBM, Chicago, IL).

\section{Results}

The data collection team approached 444 community and hospital pharmacies. Invitation to participate in this study was accepted by 431 (97.1\%) pharmacies. A total of 408 community pharmacies $(18.1 \%$ of all Jordanian community pharmacies [17]) and 23 hospital pharmacies (21.7\% of all Jordanian hospitals [18]) provided responses to the study questionnaire.

Demographics and basic characteristics of the participating pharmacies are presented in Table 1 and Table 2. The vast majority of the participating pharmacies were community pharmacies that were not part of a chain, and half of these pharmacies were run by two pharmacists. The pharmacy staff has one pharmacy assistant and one staff personnel in 130 (30.2\%) and 171 (39.7\%) of pharmacies, respectively. More than 400 (93\%) of the interviewed staff were pharmacists, and 375 (87\%) of them had the bachelor university degree, while the rest of pharmacists have a master or $\mathrm{PhD}$ degree as shown in Table 2. the median daily number of prescription 
received by pharmacies (with or without compounding orders) was 20 (IQR:10-30) while the median daily number of prescriptions containing at least one compounding order was 1.5 (IQR: $1-4.8$ ). Table 2 also shows factors that the pharmacy staff consider in deciding whether to compound prescribed medication. The main reason for requesting compounded medication is the unavailability of the required medication (196, 87.9\%), followed by lack of the needed dose among commercially available products $(152,68.2 \%)$. The main reason for not performing compounding was the absence of compounding orders in $(232,53.8 \%)$. The lack of equipment, tools, facilities, and resources required for compounding of prescriptions was reported by $24.4 \%$ of pharmacies as a reason for not compounding.

Table 3 shows data comparing pharmacies that perform compounding and pharmacies that do not compound. Being an independently-owned or chain pharmacy had no effect on its practice of compounding. The presence of a pharmacy technician (two-year diploma) as a part of the pharmacy staff did not affect the compounding practice of pharmacies. Significant differences were observed between hospital and community pharmacies in terms of compounding; community

Table 2 Characteristics of Participating Pharmacies Performing Extemporaneous Compounding

\begin{tabular}{|c|c|c|}
\hline Parameter & N (\%) & Median (IQR) \\
\hline \multicolumn{3}{|l|}{ Perform Compounding } \\
\hline Yes & $223(51.7)$ & \\
\hline Hospital & $3(1.3)$ & \\
\hline Community & $220(98.7)$ & \\
\hline Yes & $208(48.3)$ & \\
\hline Hospital & $20(9.6)$ & \\
\hline Community & $188(90.4)$ & \\
\hline Average Number of Total Prescriptions/day ( $N=382)$ & & $20(10-30)$ \\
\hline Average Number of Prescriptions per day for Compounded Orders* $(N=16)^{a}$ & & $1.5(1-4.8)$ \\
\hline Average Number of Prescriptions per week for Compounding Orders* $(N=42)^{a}$ & & $2(1-3)$ \\
\hline Average Number of Prescriptions per month for Compounding Orders* $(N=164)^{a}$ & & $2(1-4)$ \\
\hline Total Number of Years of Providing Compounding $(N=220)$ & & $10(4-18)$ \\
\hline \multicolumn{3}{|l|}{ Reasons for Providing Compounding Services $(N=223)^{b}$} \\
\hline Medication is not commercially available & $196(87.9)$ & \\
\hline Dosage Form not commercially available & $51(22.9)$ & \\
\hline Needed Dose not commercially available & $152(68.2)$ & \\
\hline Stability of the desired product & $6(2.7)$ & \\
\hline Improve adherence to medications & $102(45.7)$ & \\
\hline Other & $1(0.4)$ & \\
\hline \multicolumn{3}{|l|}{ Reasons for Not Providing Compounding Services $(N=431)^{b}$} \\
\hline No prescriptions for compounded preparations & $232(53.8)$ & \\
\hline Compounding require a lot of time (No time) & $22(5.1)$ & \\
\hline No equipment or/and supplies & $105(24.4)$ & \\
\hline Pharmacy staff lack skills/training on compounding & $15(3.5)$ & \\
\hline There are no regulations & $9(2.1)$ & \\
\hline Lack of trust on compounded medications & $23(5.3)$ & \\
\hline Compounding of medications is too expensive & $14(3.2)$ & \\
\hline Compounding of medications is too difficult & $32(7.4)$ & \\
\hline Compounded medications final cost is too high & $11(2.6)$ & \\
\hline Others & $5(1.1)$ & \\
\hline
\end{tabular}

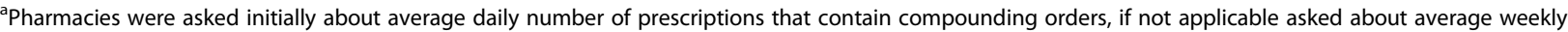
number, if not applicable about average monthly number

${ }^{b}$ Data do not sum up to $100 \%$ as multiple responses/selections were allowed

Abbreviations; IQR Interquartile Range 
Table 3 Comparison of key characteristics of compounding and non-compounding pharmacies

\begin{tabular}{|c|c|c|c|}
\hline \multicolumn{3}{|l|}{ Determinant } & \multirow{2}{*}{$\begin{array}{l}p- \\
\text { value }\end{array}$} \\
\hline Perform Compounding & Yes $(N=223)$ & No $(N=208)$ & \\
\hline & N (\%) & & \\
\hline Pharmacy Type: Hospital & $3(13)$ & $20(87)$ & $0.000^{*}$ \\
\hline Community & $220(53.9)$ & $188(46.1)$ & \\
\hline Chain & $69(60.5)$ & $45(39.5)$ & $0.623^{*}$ \\
\hline \multirow[t]{2}{*}{ Independent } & $151(51.4)$ & $143(48.6)$ & \\
\hline & Mean \pm SD & & \\
\hline Number of Pharmacists & $2.7 \pm 1.0$ & $2.1 \pm 1.8$ & $0.000^{*}$ \\
\hline Number of Assistant & $0.64 \pm 0.9$ & $0.69 \pm 0.9$ & $0.600^{*}$ \\
\hline Number of Personnel & $0.7 \pm 0.8$ & $0.5 \pm 0.7$ & $0.022^{*}$ \\
\hline Average Daily Number of All Received Prescriptions & $20(10-40)^{\#}$ & $15\left(6-27.8^{\#}\right.$ & $0.000^{*}$ \\
\hline
\end{tabular}

pharmacies were more involved in compounding. Pharmacies that prepared compounded medications had significantly higher number of pharmacists and personnel, and received, on average, a higher number of prescriptions per day.

Factors that showed significant baseline differences between compounding and non-compounding pharmacies were entered into model testing using logistic regression to test for significant association with the decision of the pharmacy to provide compounding service. As shown in Table 4, the model was significant $(\mathrm{OR}=1.47 ; p=0.000)$. The number of pharmacists $(\mathrm{OR}=1.82 ; p=0.000)$, the number of personnel $(\mathrm{OR}=1.09 ; p=0.043)$ and the average number of daily prescriptions received by the pharmacy $(\mathrm{OR}=1.02 ; p=0.002)$ were significantly associated with this service provision. While the type of pharmacy being hospital or community pharmacy and being part of a chain or not showed no association with compounding services provision.

Table 5 presents the types of compounded medications and compounding services. Dermatologist were the main prescriber specialty group requesting compounded preparations $(219,98.2 \%)$. Among the compounded dosage forms, $222(99.6 \%)$ and 204 (91.5\%), were creams and ointments, respectively, and 52 (23.3\%) were

Table 4 Analysis of Factors that Affect the Decision of Pharmacy to Perform Compounding Results Using Stepwise Logistic Regression Analysis

\begin{tabular}{llll}
\hline Determinant & \multicolumn{4}{l}{ Regression } \\
\cline { 2 - 4 } & OR & $95 \% \mathrm{Cl}$ & $p$-value \\
\hline Model & 1.47 & & 0.000 \\
Number of Pharmacists & 1.82 & $1.35-2.45$ & 0.000 \\
Number of Personnel & 1.09 & $0.72-1.65$ & 0.043 \\
Average Daily Number of All Prescriptions & 1.02 & $1.01-1.04$ & 0.002 \\
\hline
\end{tabular}

solutions. Only $5(2.2 \%)$ of the participating pharmacies that performed compounding prepared sterile products, with only $3(1.3 \%)$ of compounding pharmacies making sterile eye drops (Table 5).

About half of pharmacies have mortars, pestles, spatula, and scales, and almost one third of pharmacies have funnels, flasks, beakers, measuring cylinders, and glass rods (Table 5 ).

The main source of references for compounding protocols were the physician's order in 210 (94.2\%) of the cases, and protocols developed by the pharmacy in $100(44.8 \%)$ of the respondent pharmacies. The main source for estimation of the expiration date for compounded medications was based on pharmacist's experience $(129,57.8 \%)$ and physician's order (119, $53.4 \%)$. In 67 (30\%) of the compounding pharmacies, expiration date was not provided for compounded medications but 217 (97.3\%) of these pharmacies attach label to their compounded products. The expiration date included on the label was determined based on either in-house practice or physician's order in $149(66.8 \%)$ and $146(65.5 \%)$ of the compounding pharmacies, respectively.

In $377(75.8 \%)$ of the compounding pharmacies, no compounding records were kept, and 34 (63\%) of these records were hand-written. No pharmacy performed quality control tests of the compounded products.

\section{Discussion}

The current study addressed the prevalence, determinants, and characteristics of extemporaneous compounding and compounded products in both community and hospital pharmacies in Jordan. The present study is the first report in Jordan and one of few in the world that present the baseline data on extemporaneous compounding. Several important outcomes that 
Table 5 Compounded Products and Services Provided by Compounding Pharmacies

\begin{tabular}{ll}
\hline Parameter & Number (\%) \\
\hline Specialty of Compounding Prescriber $(N=223)^{\mathrm{a}}$ & \\
Dermatology & $219(98.2)$ \\
Pediatrics & $41(18.4)$ \\
ENT & $24(10.8)$ \\
Gastroenterology & $24(10.8)$ \\
Radiology & $11(4.9)$ \\
Other & $37(16.6)$ \\
Compounded Dosage Forms $(N=223)^{\mathrm{a}}$ & \\
Tablets & 0 \\
Capsules & 0 \\
Suppository & $1(0.4)$ \\
Ointment & $204(91.5)$ \\
Cream & $222(99.6)$ \\
Solution & $52(23.3)$ \\
Suspension & $10(4.5)$ \\
Syrup & $2(0.9)$ \\
Paste & $9(4)$ \\
Powder & $3(1.3)$ \\
Yompounded Sterile Products $(N=223)$ & \\
No & $5(2.2)$ \\
Y & $218(97.8)$
\end{tabular}

Types of Compounded Sterile Products $(N=223)$

Eye Drops

$3(1.3)$

Electrolytes

Total Parenteral Nutrition

$1(0.4)$

$2(0.9)$

Chemotherapy

$2(0.9)$

Equipment and Tools Used for Compounding Availability $(N=431)^{a}$

$\begin{array}{ll}\text { Mortars } & 214(49.7) \\ \text { Pestles } & 213(49.4) \\ \text { Capsule Machines } & 1(0.2) \\ \text { Ointment Mill } & 14(3.2) \\ \text { Tablet Machines } & 0 \\ \text { Suppositories Molds } & 7(1.6) \\ \text { Beaker } & 131(30.4) \\ \text { Funnel } & 125(29) \\ \text { Spatula } & 213(49.4) \\ \text { Scale } & 188(43.6) \\ \text { Flask } & 118(27.4) \\ \text { Water Bath } & 31(7.2) \\ \text { Measuring Cylinder } & 114(26.5) \\ \text { Glass Rod } & 137(31.8) \\ \text { Ointment Slab } & 60(13.9)\end{array}$

Sources for protocols of Compounded Medications $(N=223)^{a}$
Table 5 Compounded Products and Services Provided by Compounding Pharmacies (Continued)

\begin{tabular}{ll}
\hline Parameter & Number (\%) \\
\hline Published Literature & $5(2.2)$ \\
USP & $9(4)$ \\
BP & $25(11.2)$ \\
Self-Reports & $100(44.8)$ \\
Other Pharmacies or Hospitals & $11(4.9)$ \\
Prescriber/Physician & $210(94.2)$ \\
Provision of Expiration Date on Compounded Medications & $(N=431)$ \\
Yes & $156(70)$ \\
No & $67(30)$
\end{tabular}

Sources for Estimation of Expiration Date for Compounded Medication $(N=223)^{\mathrm{a}}$

$\begin{array}{ll}\text { Published Literature } & 8(3.6) \\ \text { USP } & 6(2.7) \\ \text { BP } & 16(7.2) \\ \text { Self-Reports } & 129(57.8) \\ \text { Other Pharmacies or Hospitals } & 7(3.1) \\ \text { Prescriber/Physician } & 119(53.4) \\ \text { Label Provided on Compounded Medication }(N=223) & \\ \text { Yes } & 217(97.3) \\ \text { No } & 6(2.7)\end{array}$

References for Information Provided on the Label of Compounded Medication $(N=223)$

$\begin{array}{ll}\text { Published Literature } & 0 \\ \text { USP } & 36(16.1) \\ \text { BP } & 11(4.9) \\ \text { Pharmacist's Experience } & 149(66.8) \\ \text { Prescriber/Physician } & 146(65.5)\end{array}$

Records for Compounded Medications ( $N=431)$

Yes

No

$377(75.8)$

Type of Record/Log-book ( $N=54)$

Electronic 20 (37)

Hand-written 34 (63)

esting of Quality of Compounded Medications ( $N=223) \quad 0$

Most Frequently Used Expiration Date, Weeks $(N=223) \quad 4(0.9)$

${ }^{a}$ Data do not sum up to $100 \%$ as multiple responses/selections were allowed

emerged from this study can be used in the improvement of pharmacy practice.

The high prevalence of the compounding practice in community pharmacies emphasizes the need of proper education and training of pharmacy students in extemporaneous compounding. There is also a clear need to incorporate extemporaneous compounding in professional development and continued education programs offered by the Jordanian Pharmacy Syndicate and the 
Jordanian Pharmacy Board. Results from this study also show the critical need for implementation of quality testing of compounded products and standardized regulations to ensure safe compounding practices.

Data from this study show that $52 \%$ of Jordanian pharmacies perform compounding which is less than the percentage (add this percentage) reported in USA [3, 19], The percentage of prescriptions received per day that contain at least one compounding order is higher than those reported in literature $[3,6,16,19]$. These differences may be attributed to the fact that this study involved all types of Jordanian pharmacies (large and small, and hospital and community pharmacies). Buurma and colleagues (2003), reported a decline in Dutch compounding pharmacies and a rise of specialized firms that compound and promote those preparations that are most broadly needed [16].

Several factors can play a role in the pharmacy's decision to offer compounding services. These factors include the availability of the staff (both pharmacists and personnel) and the number of all prescriptions received and filled by the pharmacy. Pharmacies with a higher number of pharmacists reported making more compounded preparations. The time and efforts required to develop and manage extemporaneous compounding service are critical factors that influence the prevalence of compounding in Jordanian pharmacies. This issue has been addressed in Dutch community pharmacies by preparing a large part of compounded medications from semi-manufactured or almost finished products provided by specialized firms. Such products require minimal work to be finished at the pharmacy [16].

As would be expected, the number of all prescriptions filled by the pharmacy correlates directly with the compounding services. However, some pharmacies with a long tradition in providing compounding services were observed to have a disproportionately higher number of compounding order to their overall number of prescriptions filled daily.

Dermatologists were reported to be the prescribers of compounding orders by $98.2 \%$ of the compounding pharmacies in Jordan. They were ahead of other specialties by a large margin. This is in agreement with data from previously reported study on top indications for extemporaneously compounded products [16] and could be related to the observation that creams and ointments were the most commonly compounded dosage forms by compounding pharmacies.

The fact that community pharmacies were more involved extemporaneous compounding could be understood in the context that most of the compounded dosage forms are creams and ointments and the main prescriber specialty group was "Dermatologists". Most health complaints addressed by dermatologists do not require hospitalization and prescriptions are often filled by community rather than hospital pharmacies.

Pediatricians were the second highest specialists in terms of requesting compounded products with $18.4 \%$ of the compounding pharmacies reporting receiving orders for compounding from pediatricians. Buurma and colleagues reported that patients less than 12 years old received more extemporaneously prepared medications than other age groups [16].

Solutions were the third most commonly compounded dosage form (23.3\%) in Jordanian compounding pharmacies.

Previously reported studies showed that patientcentered pharmaceutical care is the main reason for pharmacists to provide compounded medications $[3,6]$. Pharmaceutical care should address all patients needs by ensuring the availability of all needed medications, including ingredients combinations, dosage forms, and doses that are patient-specific. Individualized patient care results in improved patient's adherence to medications and consequently better therapeutic outcomes and quality of life.

As shown in this study, the main reasons for requesting and providing compounding services is the unavailability of the required medications or a specific dosage form $(87.9 \%)$ and the unavailability of the required dose (68.2\%). An earlier study has linked the reasons for compounding with the specialty of the prescriber with dermatologists being reported to order combinations that are usually unavailable commercially and pediatricians requiring patients-tailored dosage strengths [16].

The majority of pharmacies participating in this study stated lack of prescriptions for extemporaneously compounded products as a key reason for not providing compounding services. Zaid et al. [6] and McPherson et al. [3] also reported that that the main reason for pharmacists being non-compounders is not receiving prescriptions. Surprisingly, the second reported reason for not compounding was the lack of equipment and supplies. This is unexpected because by the law, each licensed pharmacy is required to have compounding tools and equipment. Interestingly, required time, cost, and difficulty of preparing these medications did not have impact on the pharmacy's decision to provide compounding services.

In general, sterile compounding was not performed in pharmacies participating in this study. This can be explained by the fact that compounding of sterile products is expensive because it requires sterile environment, specific procedures and equipment. Improper preparation of sterile compounded preparations may easily lead to contaminated product [6].

Buurma and colleagues reported that in $42 \%$ of the cases, pharmacies did not use a standardized or semi- 
standardized protocol [16]. The current study showed the lack of standardized procedures for compounding with vast majority of the respondents stating that the physician order is the source of the procedure while $44.8 \%$ reported the use of procedures developed in the pharmacy for compounding different products. The same resources were reported to be relied upon for estimation of expiration date for compounded medicines and labeling of the compounded products.

Moreover, the study uncovers the lack of documentation and quality assurance of the compounded product, although $24.2 \%$ of pharmacies keep records for their products and considerable number of pharmacists seeks patients' feedback on their products. Despite of all of this, there is a need for agreed on protocols that cover procedures, labelling including expiry date, and quality control tests that should be followed and guaranteed during extemporaneous compounding to assure effectiveness and quality of the final product and improve the physicians as well as patients' confidence in these products.

The main limitation of this study is the difference in the number of community (408) and hospital pharmacies (23) included in the study. However, it is worth noting that we tried to include a similar proportion of community (18.1\%) and hospital (21.7\%) pharmacies of all those operating in Jordan. Another limitation is the fact that this study did not cover the compounding practice from the perspective of prescribers and patients. This includes the study of drivers for prescribing compounded products and the patients' experience with the compounding services offered by pharmacies. We are attempting to cover this aspect in a future study.

The data presented in this study was presented to and discussed with faculty members at the School of Pharmacy, University of Jordan in order to modify curriculum to better cover procedures, labelling including expiry date, and quality control tests that should be followed and guaranteed during extemporaneous compounding to assure effectiveness and quality of the final product and improve the physicians as well as patients' confidence in these products. In addition, a plan was developed for offering a continuing education program to practicing pharmacists in collaboration with the Jordanian Pharmacists Syndicate in the area of best practices in extemporaneous compounding.

\section{Conclusion}

Extemporaneous compounding is a widely spread pharmaceutical practice among community pharmacies in Jordan. Ointments and creams are the most commonly prescribed and compounded extemporaneous products. A higher number of pharmacists, pharmacy personnel and the total number of prescriptions were significantly associated with a higher number of extemporaneous products compounded. The lack of standardized procedures for preparing extemporaneous products, scientific basis for determination of expiration date of compounded products, and lack of quality control testing prompts an action.

\section{Supplementary information}

Supplementary information accompanies this paper at https://doi.org/10. 1186/s12913-019-4684-y.

Additional file 1. Extemporaneous Compounding In Jordanian

Pharmacies Survey

\section{Abbreviations}

IRB: Institutional Review Board; KMO: Kaiser-Meyer-Olkin; P: prevalence values; PCA: Principal Components Analysis; PI: principal investigator; SP-UJ: School of Pharmacy - University of Jordan; USP: United States Pharmacopeia

\section{Acknowledgments}

The authors would like to thank Miss Lujain Alhiary, Miss Roaa Alshogirat, Miss Sabreen Alqwabah, and Mr. Momen Durrah; the School of Pharmacy at the university of Jordan graduates, for participating in data collection.

\section{Consent for publications}

Not applicable.

\section{Authors' contributions}

HA participated in designing the study, revised the data collection tool and participated in the writing of the manuscript. SJ and NM developed the data collection tool, participated in the data collection and data entry. AR revised and modified the data collection tool and the written manuscript. EE revised the data analysis and participated in writing the manuscript. SA participated in designing the study, revised the data collection tool, carried out the data analysis, and participated in the writing of the manuscript. All authors have read and approved the manuscript. In addition, all authors are aware of this submission and agree with it.

\section{Funding}

This research was supported by The Deanship of Academic Research at the University of Jordan (2158). The funding institution did not participate in the design of the study and collection, analysis, and interpretation of data and in writing the manuscript.

\section{Availability of data and materials}

The datasets used and analyzed during the current study are available from the corresponding author on reasonable request.

\section{Ethics approval and consent to participate}

Consent was given in writing at the beginning of the interview.

This study was approved by School of Pharmacy - University of Jordan (SPUJ) Scientific Research Committee and the Clinical Institutional Review Board Committee affiliated with the Jordan University Hospital (IRB: 7/2018).

\section{Competing interests}

The authors declare that they have no competing interests.

\section{Author details}

'Department of Pharmaceutics and Pharmaceutical Technology, School of Pharmacy, The University of Jordan, Queen Rania Street, Amman 11942 Jordan. ${ }^{2}$ Wegmans School of Pharmacy, St. John Fisher College, Rochester, NY, USA. ${ }^{3}$ Department of Biopharmaceutics and Clinical Pharmacy, School of Pharmacy, The University of Jordan, Amman, Jordan. 
Received: 5 September 2019 Accepted: 27 October 2019

Published online: 08 November 2019

\section{References}

1. Falconer JR, Steadman KJ. Extemporaneously compounded medicines. Aust Prescr. 2017:40(1):5-8.

2. Minghetti P, Pantano D, Gennari CG, Casiraghi A. Regulatory framework of pharmaceutical compounding and actual developments of legislation in Europe. Health Policy. 2014;117(3):328-33.

3. McPherson TB, Fontane PE, Jackson KD, Martin KS, Berry T, Chereson R, Bilger R. Prevalence of compounding in independent community pharmacy practice. J Am Pharm Assoc. 2006:46(5):568-73.

4. Treadway AK, Craddock D, Leff R. Practices of pharmacies that compound extemporaneous formulations. Am J Health Syst Pharm. 2007;64(13):1403-9.

5. Giam JA, McLachlan AJ, Krass I. Community pharmacy compounding-impact on professional status. Int J Clin Pharm. 2011;33(2):177-82.

6. Zaid AN, Al-Ramahi R, Shahed Q, Saleh B, Elaraj J. Determinants and frequency of pharmaceutical compounding in pharmacy practice in Palestine. Int J Pharm Pract. 2012;20(1):9-14.

7. Wiedyaningsih C, Kristina SA, Widyakusuma NN, Aditama H. Opinion and expectation of pharmacists on providing extemporaneous compounding in jogjakarta and central java provinces. Indonesia Int J Pharm Pharm Sci. 2017; 9(7):79-82.

8. Taylor S, Hays C, Glass B. Compounding medications in a rural setting: an interprofessional perspective. J Multidiscip Healthc. 2018;11:197-204.

9. Dooms M, Carvalho M. Compounded medication for patients with rare diseases. Orphanet J Rare Dis. 2018;13(1):1.

10. Nahata MC. Pediatric drug formulations: challenges and potential solutions. Ann Pharmacother. 1999;33(2):247-9.

11. Brion F, Nunn AJ, Rieutord A. Extemporaneous (magistral) preparation of oral medicines for children in European hospitals. Acta Paediatr. 2003;92(4): 486-90.

12. Balan S, Hassali MA, Mak VSL. Challenges in pediatric drug use: a pharmacist point of view. Res Social Adm Pharm. 2017;13(3):653-5.

13. FDA Consumer Health Information, The Special Risks of Pharmacy Compounding. 2007. https://www.pharmwatch.org/reports/compounding_ risks.pdf .

14. The United States pharmacopeia, 39th rev., and the national formulary, 34th ed. Rockville, MD: United States Pharmacopeial Convention; 2016.

15. Gudeman J, Jozwiakowski M, Chollet J, Randell M. Potential risks of pharmacy compounding. Drugs R D. 2013 Mar;13(1):1-8.

16. Buurma $H$, de Smet PA, van den Hoff OP, Sysling H, Storimans M, Egberts AC. Frequency, nature and determinants of pharmacy compounded medicines in Dutch community pharmacies. Pharm World Sci. 2003;25(6): 280-7.

17. Jordan Pharmacists Association official website http://jpa.org.jo/page/ pharmacy-profession-jordan .

18. Ministry of Health, Jordan official website, http://apps.moh.gov.jo/MOH/ Files/Publication/PDF2011_1.pdf

19. Schommer JC, et al. Prescription compounding in Wisconsin pharmacies. Wisc Pharm. 1990;59:24-5.

\section{Publisher's Note}

Springer Nature remains neutral with regard to jurisdictional claims in published maps and institutional affiliations.

Ready to submit your research? Choose BMC and benefit from:

- fast, convenient online submission

- thorough peer review by experienced researchers in your field

- rapid publication on acceptance

- support for research data, including large and complex data types

- gold Open Access which fosters wider collaboration and increased citations

- maximum visibility for your research: over $100 \mathrm{M}$ website views per year

At BMC, research is always in progress.

Learn more biomedcentral.com/submissions 